\title{
Note: A silicon-on-insulator microelectromechanical systems probe scanner for on-chip atomic force microscopy
}

\author{
Anthony G. Fowler, Mohammad Maroufi, and S. O. Reza Moheimania) \\ School of Electrical Engineering and Computer Science, University of Newcastle, \\ Callaghan, NSW 2308, Australia
}

(Received 5 February 2015; accepted 9 April 2015; published online 21 April 2015)

\begin{abstract}
A new microelectromechanical systems-based 2-degree-of-freedom (DoF) scanner with an integrated cantilever for on-chip atomic force microscopy (AFM) is presented. The silicon cantilever features a layer of piezoelectric material to facilitate its use for tapping mode AFM and enable simultaneous deflection sensing. Electrostatic actuators and electrothermal sensors are used to accurately position the cantilever within the $\mathrm{x}-\mathrm{y}$ plane. Experimental testing shows that the cantilever is able to be scanned over a $10 \mu \mathrm{m} \times 10 \mu \mathrm{m}$ window and that the cantilever achieves a peak-to-peak deflection greater than $400 \mathrm{~nm}$ when excited at its resonance frequency of approximately $62 \mathrm{kHz}$. (C) 2015 AIP Publishing LLC. [http://dx.doi.org/10.1063/1.4918729]
\end{abstract}

The atomic force microscope (AFM) has proven to be an invaluable tool for researchers working in a wide range of nanotechnology related fields. First introduced in $1986,{ }^{1}$ the AFM is a mechanical microscope that is able to interrogate the surface of physical samples to produce 3D images with atomic-scale resolution.

The AFM typically features a cantilever with a sharp probe tip of a few nanometers in diameter. A nanopositioner is used to scan the probe across the complete surface of a sample, allowing the topography of the sample to be mapped in three dimensions using the deflection measurements of the cantilever and the lateral position of the nanopositioner.

The continued development of the AFM since its inception has resulted in a range of commercial systems that are able to perform high-speed scans with sub-nanometer resolution. However, such devices continue to be based on conventional macroscale technologies, with their high purchase costs generally prohibiting them from seeing widespread use outside of specialized scientific research applications.

Ongoing research has demonstrated that microelectromechanical systems (MEMS) represent a viable platform for the development of novel nanopositioning devices. ${ }^{2,3}$ In addition to possessing performance that is comparable with that of typical macroscale devices, the use of MEMS processes for this application introduces a number of other significant advantages that include a much smaller packaged size and greatly reduced costs for batch fabrication. ${ }^{4}$ With the nanopositioning system being one of the major components of the AFM, the development of a MEMS-based alternative represents a large step towards potentially creating a complete AFM that is fully fabricated using MEMS technology. Such a device would be much more cost-effective and portable than conventional systems, potentially expanding its use within existing scientific applications and allowing it to be introduced into a range of new roles.
Our previous research to date exploring the use of MEMS nanopositioners for AFM has involved demonstrating scans performed in open loop ${ }^{5}$ and closed loop with both raster ${ }^{6,7}$ and non-raster trajectories. ${ }^{8}$ However, whereas these previous results used the cantilever of a conventional AFM to obtain the deflection data used to generate the obtained images, this paper presents a new 2-degree-of-freedom (DoF) MEMSbased probe scanner that features an integrated piezoelectrically actuated cantilever suitable for directly measuring the topography of a sample. This is a significant step towards the complete replacement of a conventional macroscale AFM with a fully microfabricated system.

Scanning electron microscope (SEM) images of the MEMS probe scanner are shown in Fig. 1. The MEMS device is fabricated using MEMSCAP's PiezoMUMPs process,${ }^{9}$ which features a $10 \mu \mathrm{m}$ silicon device layer and a $0.5 \mu \mathrm{m}$ thick layer of aluminum nitride (AlN) as the piezoelectric material. A $2165 \mu \mathrm{m} \times 650 \mu \mathrm{m}$ stage is located at the center of the device, while a two-section cantilever with a resonance frequency of approximately $63 \mathrm{kHz}$ is designed on one side of the stage. The chosen resonance frequency is comparable with commercially available cantilevers for tapping mode AFMs. The cantilever has a total length of $640 \mu \mathrm{m}$ and a width of $40 \mu \mathrm{m}$ and $240 \mu \mathrm{m}$ at the narrow and wide sections, respectively. A layer of piezoelectric material is deposited on both sections of the cantilever for out-of-plane actuation.

To move the stage in two perpendicular directions, electrostatic comb-drive actuators are used. The mass of the comb structures is reduced while maintaining their mechanical stiffness through the use of a trapezoidal geometry. ${ }^{8}$ Two sets of comb drives are implemented on each side of the stage in the $\mathrm{x}$ direction. For each set, the actuation force is transferred to the stage using a shuttle beam via two tethering beams. Through the inclusion of electrostatic actuators on both sides of the stage in the $\mathrm{x}$ direction, bidirectional displacements as well as the implementation of a linear actuation mechanism are possible. ${ }^{7}$ Similarly, in the y direction, a shuttle beam and ten tethering beams are used to connect the actuators to the

$\overline{\text { a) Reza.Moheimani@newcastle.edu.au }}$ 


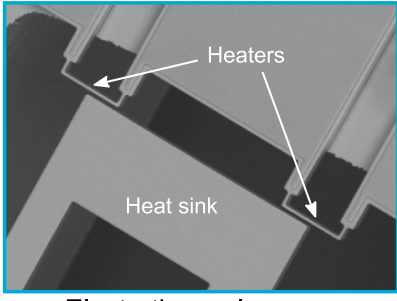

Electrothermal sensor

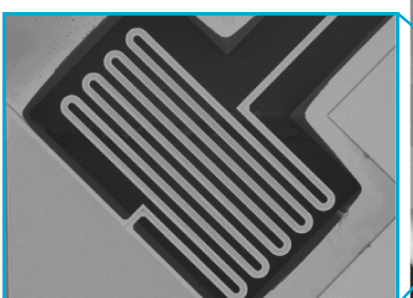

Serpentine flexure with metal layer for connection to AIN

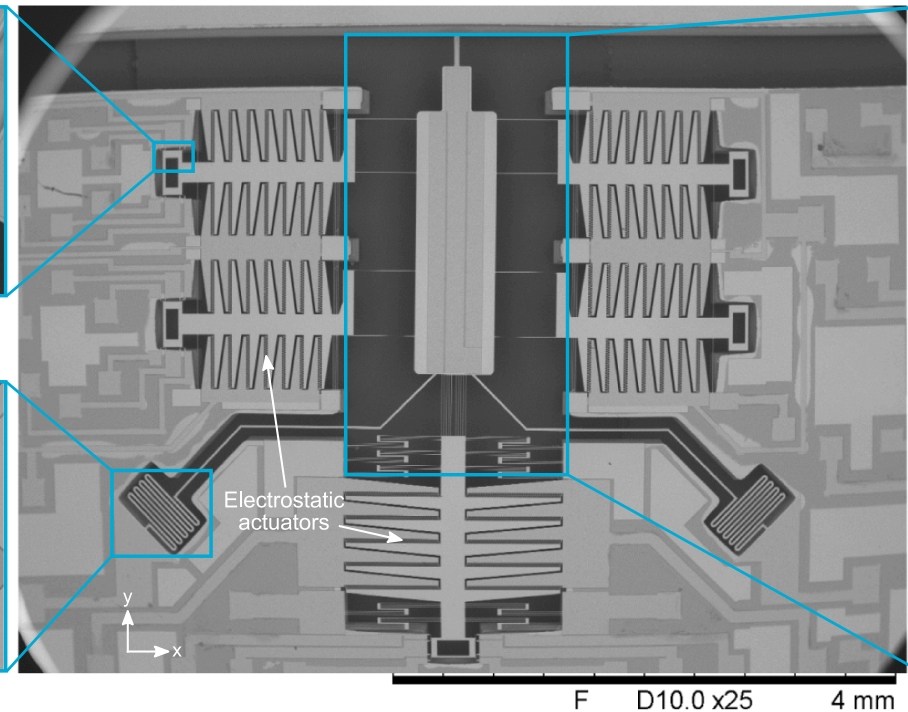

$4 \mathrm{~mm}$

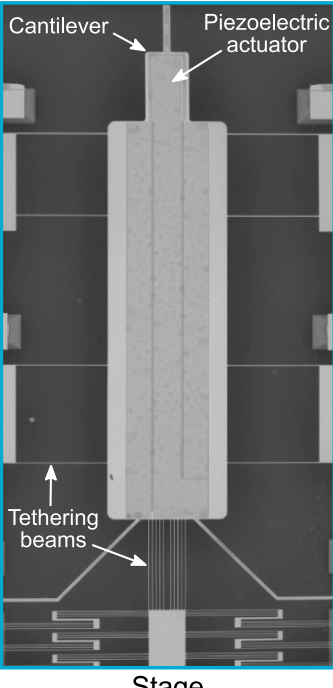

Stage

FIG. 1. SEM images of the fabricated MEMS probe scanner, including close-up images of the device's main mechanical components.

stage. The mechanical suspension system in this device largely consists of clamped-clamped beams connected to the shuttle beam. However, due to the unidirectional displacement of the stage along the y axis, folded flexures are used to improve the linearity of the device in this direction. ${ }^{10}$

To measure the stage displacement in each direction, electrothermal sensors with straight beam heaters are incorporated. The integration of on-chip sensors means that an appropriate controller can be implemented to improve the positioning accuracy of the device. In addition, the incorporation of two sensors in the $\mathrm{x}$ direction makes the measurement of potential in-plane rotations of the scan table viable. Possible rotations can also be eliminated by using the four sets of actuators in the $\mathrm{x}$ direction in a control loop.

The fabrication process provides an additional oxide layer with an aluminum layer on top, which is used to provide signals to the piezoelectric layer on the cantilever. However, a wide silicon structure is needed for routing to the piezoelectric layer due to masking tolerances. Beam structures with $18 \mu \mathrm{m}$ width are used for signal routing, and to reduce their effect on the stage's dynamics, serpentine structures are incorporated as the anchoring structures.

By dimensional tuning using a finite element model (FEM), the first and second modes of the device are designed to be in-plane. As shown in Fig. 2, the FEM (constructed in CoventorWare) predicts that the first two resonant modes are located at $2.83 \mathrm{kHz}$ in the $\mathrm{y}$ direction and $2.91 \mathrm{kHz}$ in the $\mathrm{x}$ direction.

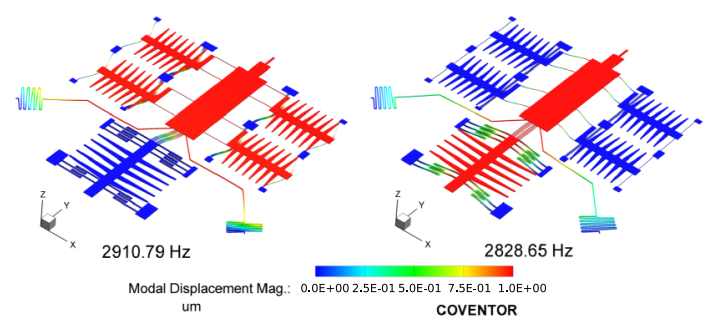

FIG. 2. Modal analysis of the device using FEM. Left: $x$ direction. Right: $y$ direction.
The fabricated device was experimentally characterized, with Fig. 3 showing the frequency response of the system along the $\mathrm{x}$ and $\mathrm{y}$ axes as measured by a Polytec MSA-050-3D Micro-System Analyzer (MSA). These results indicate that the first resonance modes of the device are located at $2.94 \mathrm{kHz}$ and $2.65 \mathrm{kHz}$, which correspond relatively well to the simulated modal frequencies. These frequency responses were measured using both the MSA and the device's integrated electrothermal sensors, and the close match between the two responses at all frequencies up to twice that of the primary resonance indicates that the sensors are able to satisfactorily capture the dynamics of the nanopositioner.

The $\mathrm{x}$-axis actuators on both sides of the stage are incorporated in a linear actuation configuration with a $35 \mathrm{~V}$ DC bias. Fig. 4(a) shows the displacement of the stage (measured using the MSA) and the corresponding sensor output when a differential actuating signal is applied to the $\mathrm{x}$-axis actuator. These results indicate that a total displacement range greater than $10 \mu \mathrm{m}$ is achievable for the $\mathrm{x}$ axis and that the corresponding sensor calibration factor is approximately $1.40 \mathrm{~V} / \mu \mathrm{m}$. The corresponding results for the y axis are shown in Fig. 4(b),

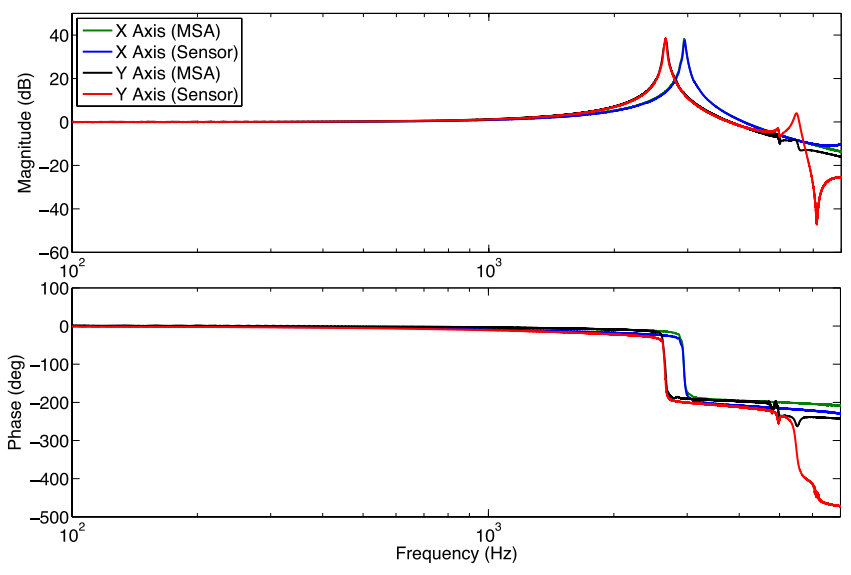

FIG. 3. Frequency response of fabricated device, obtained using MSA and electrothermal sensors. 


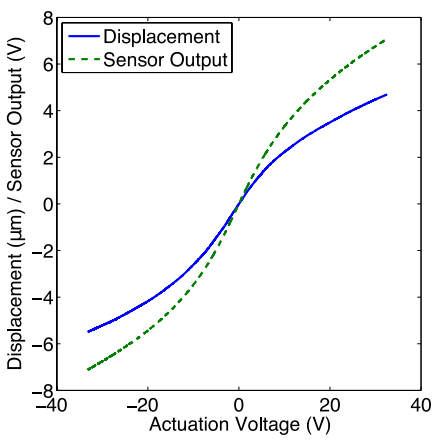

(a)

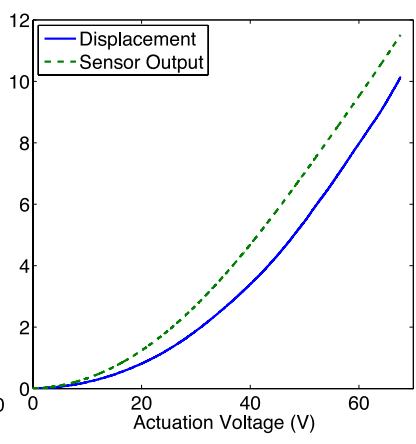

(b)
FIG. 4. Displacement and sensor output vs. actuation voltage. (a) $\mathrm{x}$ axis. (b) y axis.

which indicates a stroke of approximately $10 \mu \mathrm{m}$ and a sensor calibration factor of $1.17 \mathrm{~V} / \mu \mathrm{m}$.

The cross coupling between the two axes of the device was determined by actuating each axis over its full range and measuring the stage displacement along the orthogonal axis. These results show acceptable cross coupling of $-26.1 \mathrm{~dB}$ from the $\mathrm{x}$ to $\mathrm{y}$ axes and $-34.8 \mathrm{~dB}$ from the $\mathrm{y}$ to $\mathrm{x}$ axes.

The frequency response of the cantilever was measured by applying a wideband chirp signal to the piezoelectric actuator and measuring the out-of-plane deflection of the cantilever using a Polytec MSA-400. This shows that the dominant resonance mode of the cantilever is located at $61.98 \mathrm{kHz}$, which matches closely with a performed FEM simulation. Fig. 5(a) shows the mode shape of the cantilever at this frequency as measured by the MSA-400, confirming that this is an out-of-plane vibrational mode that is suitable for tapping mode AFM. When this mode is excited by applying a $1 \mathrm{~V}$ sinusoidal signal at the identified resonance frequency, the cantilever produces a sinusoidal oscillation with a peak-topeak displacement in excess of $400 \mathrm{~nm}$, as shown in Fig. 5(b).

With these tests demonstrating the cantilever's satisfactory characteristics, a probe tip will be added to the end of the cantilever in a future revision of the device that will enable the imaging of samples to be performed. The feasibility of monolithically fabricating a suitable tip together with the rest of the device is currently being investigated, with potential methods including anisotropically etching the silicon cantilever to create a sharp pyramidal structure. ${ }^{11}$

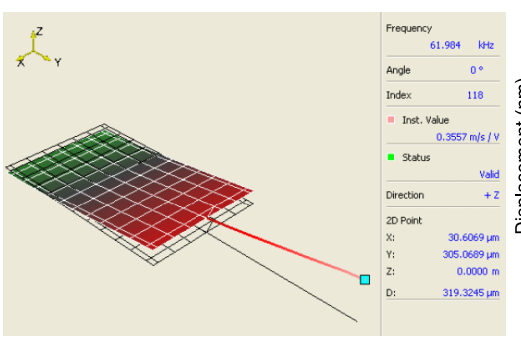

(a)

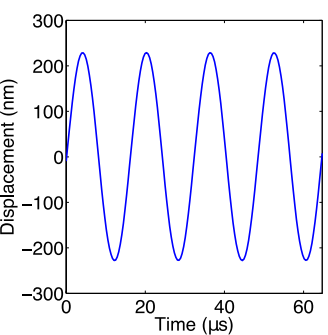

(b)
FIG. 5. (a) Mode shape of cantilever's dominant resonance mode. (b) Outof-plane displacement of cantilever due to a sinusoidal excitation.

In summary, a new MEMS-based probe scanner for onchip AFM has been demonstrated, comprising a 2-DoF nanopositioner with an integrated silicon cantilever. The nanopositioner is able to scan the probe over a $10 \mu \mathrm{m} \times 10 \mu \mathrm{m}$ window with a bandwidth greater than $2.6 \mathrm{kHz}$ along each axis, and testing of the piezoelectrically actuated cantilever confirms its suitability for tapping mode AFM. Through future developments that may involve the addition of a sharp tip to the cantilever and the implementation of a self-sensing technique, ${ }^{12}$ this device provides a valuable platform for the development of a complete MEMS on-chip AFM.

${ }^{1}$ G. Binnig, C. F. Quate, and C. Gerber, Phys. Rev. Lett. 56, 930 (1986).

${ }^{2}$ M. Azizi, N. Sarkar, and R. R. Mansour, IEEE Trans. Microwave Theory Technol. 61, 4621 (2013).

${ }^{3}$ Y.-S. Kim, N. G. Dagalakis, and S. K. Gupta, J. Micromech. Microeng. 24, 075009 (2014).

${ }^{4}$ S. Bergna, J. J. Gorman, and N. G. Dagalakis, in 2005 ASME International Mechanical Engineering Congress and Exposition (ASME, Orlando, USA, 2005), pp. 1-8.

${ }^{5}$ A. G. Fowler, A. N. Laskovski, A. C. Hammond, and S. O. R. Moheimani, J. Microelectromech. Syst. 21, 771 (2012).

${ }^{6}$ A. Mohammadi, A. G. Fowler, Y. K. Yong, and S. O. R. Moheimani, J. Microelectromech. Syst. 23, 610 (2014).

${ }^{7}$ M. Maroufi, A. Bazaei, and S. O. R. Moheimani, IEEE Trans. Control Syst. Technnol. 23, 504 (2015).

${ }^{8}$ M. Maroufi, A. G. Fowler, A. Bazaei, and S. O. R. Moheimani, Rev. Sci. Instrum. 86, 023705 (2015)

${ }^{9}$ A. Cowen, G. Hames, K. Glukh, and B. Hardy, PiezoMUMPs Design Handbook (MEMSCAP Inc., 2014).

${ }^{10}$ R. Legtenberg, A. W. Groeneveld, and M. Elwenspoek, J. Micromech. Microeng. 6, 320 (1996).

${ }^{11}$ Q. Yu, G. Qin, C. Darne, C. Cai, W. Wosik, and S.-S. Pei, Sens. Actuators, A 126, 369 (2006).

${ }^{12}$ M. G. Ruppert and S. O. R. Moheimani, Rev. Sci. Instrum. 84, 125006 (2013). 